\title{
Evaluation of learning of asymmetrical bimanual tasks and transfer to converse pattern: Load, temporal and spatial asymmetry of hand movements
}

\author{
Mohammadreza Doustan ${ }^{1, *}$, Mehdi Namazizadeh², Mahmoud Sheikh², and Naser Naghdi ${ }^{3}$ \\ ${ }^{1}$ Faculty of Sport Sciences, Shahid Chamran University of Ahvaz, Ahvaz, Iran; ${ }^{2}$ Faculty of Sport Sciences, \\ Tehran University, Tehran, Iran; and ${ }^{3}$ Department of Neurology, Iran Pasteur Institute, Tehran, Iran
}

Copyright: (C) 2019 M. Doustan et al. This is an open access article licensed under the Creative Commons Attribution License (https://creativecommons.org/licenses/by/4.0/).

\begin{abstract}
Background: In most daily activities, we are required to use both hands. In many motor skills like playing guitar, the left and right hand must perform asymmetric movements with different timing. Objective: The aim of the study was to evaluate the effect of learning in various asymmetrical bimanual tasks and to evaluate the transfer to tasks with converse hand movements. Methods: Thirty right-handed male students (age $21.5 \pm 1.3$ years) who had no motor disorders were divided into three groups. Participants of each group were trained for four days after a pretest. All participants performed asymmetrical bimanual drawing of a circle with each hand. Participants in the first group differed in terms of load in each hand, those in the second group differed in the speed of hand movement and those in the third group differed in the range of motion. The test was carried out in simultaneous bimanual movement both as practiced (learning acquisition test) and substitution of patterns between the two hands (transfer test). To analyze the data, repeated measures analysis of variance was performed. Results: For the acquisition test, significant differences were found between the results of the pretest, the posttest, and the retention test across all three groups. In terms of the transfer test, the first group showed a significantly better performance than their performance on the acquisition $(p=.001)$. No such differences were found between the performance of the second group on the two tests $(p=.945)$. Finally, the third group performed significantly better on the transfer test than on the acquisition test. $(p=.047)$. Conclusions: The present study found similar effects of motor learning on various asymmetrical bimanual motor tasks, but different inter-group performance on learning and transfer tasks.
\end{abstract}

Keywords: bimanual coordination, hierarchical control, effector-independent hypothesis

\section{Introduction}

Some of the daily motor skills involve precise timing for using both hands. Most of these skills such as opening a bottle, typing, or playing the guitar involve using the two hands differently. A good majority of studies have revealed that doing two different tasks with the two hands, when each hand performs a different action simultaneously, incurs bilateral interference even when each hand can perform separately without difficulty (Klapp, Nelson, \& Jagacinski, 1998; Kurtz \& Lee, 2003). These observations make it clear that the central nervous system (CNS) can control symmetric bimanual movements without any problem while it is

\footnotetext{
* Address for correspondence: Mohammadreza Doustan, Department of Motor Behavior, Faculty of Sport Sciences, Shahid Chamran University of Ahvaz, Golestan Blvd., Ahvaz, 6135783151 Iran. E-mail: m.doustan@scu.ac.ir
}

deficient and constrained in asymmetric movements, indicating the high tendency of the CNS toward producing symmetric or similar movements (Swinnen, 2002; Swinnen, Dounskaia, \& Duysens, 2002). This shows that bimanual skills have a different method of control from that of unimanual movements, especially when each part of the body simultaneously makes a different movement temporally and spatially (Vangheluwe, Suy, Wenderoth, \& Swinnen, 2006).

Regarding how bimanual movements are controlled by the CNS, there have been various theories in whose interpretation of these movements there are critical contradictions and differences. The intense tendency of the two hands toward producing similar movements in terms of time and space features in bimanual movements results in the theory that there is a single motor program for both hands (Schmidt \& Lee, 1999).

Generalized Motor Program (GMP) theory assumes that the motor representation is independent 
of the effector, implying that a motor skill whose motor program has been acquired can be performed by different parts of the body (Marteniuk \& MacKenzie, 1980). It also expresses that even when each hand is performing a different action simultaneously, just one motor program controls the movement, although the specific parameters of each part of the body have been defined independently.

The other useful theory about coordination is that it is created in multiple levels, from perceptual-cognitive (higher levels) to nervous-muscular (lower levels); that is coordination is achieved in bimanual activities hierarchically and in fact it consists of a perceptual-cognitive level, a nervous-muscular level and effector-specific level (Swinnen \& Carson, 2002; Swinnen, Jardian, Meulenbroek, Dounskaia, \& Hofkens-Van Den Brandt, 1997). Coordination is the result of the interaction between several parts of CNS including the perceptualcognitive level, nervous-muscular level and effector-specific level (Schmidt \& Lee, 1999). Dynamic systems approach delineates both the behavior and the brain basic performance in a similar framework (Bangert, Reuter-Lorenz, Walsh, Schachter, \& Seidler, 2010).

The effector-independent motor program in bimanual coordination movements proposed in GMP theory is the issue occasionally challenged by other theoretical models. The approach, empirically evaluating the independence of effector from motor memory and other theoretical models of bimanual coordination, studies transfer in which a new motor skill is performed by an effector system trained before and subsequently a similar task is performed by another effector system which has not been trained yet. Positive inter-limb transfer refers to the issue that prior experience of performing a skill by an effector system, positively affects its performing by a new effector system. Therefore, it refers to the independence of effector from motor memory. On the contrary, negative transfer denotes that performing a similar skill by another effector system interferes with its prior experience, thus, on no account can independence of the effector be affirmed (Vangheluwe et al., 2006). Regarding neurology, some researchers (Walsh, Small, Chen, \& Solodkin, 2008; Wu, Wang, Hallett, Li, \& Chan, 2010) have stated that the dominant cerebral hemisphere is responsible for activating and leading bimanual movement.

The researches on unimanual transfer show a positive transfer from one limb to another, while the findings regarding the bimanual movements have revealed contradictory results. Zanone and Kelso (1992, 1997) proved that a bimanual movement involving the fingers' opening and closing is transferred to its symmetric pair. Zanone and Kelso (1997) also showed a positive transfer from arms to legs and vice versa. This positive transfer results in the intrinsic independence of effector system from memory representation in bimanual tasks. These results support the generalized motor program theory. Nevertheless, Vangheluwe et al. (2006) introduced hard evidence concerning the negative transfer from prior learning of bimanual coordination task to asymmetric coupling condition. Employing a pattern in which the left hand swirled two times faster than the right hand, Vangheluwe et al. (2006) also investigated transfer to its converse pattern that is, this time, the right hand swirled two times faster than the left hand. The results revealed a negative transfer. This transfer led to reconsideration of motor representation confirming the concept of effector-independence. In addition, Sisti et al. (2011) mentioned that with an increase the relative angular velocity between the two hands, the bimanual action deteriorated. This indicates the speed differences in the movement of the two hands is an influential factor in bimanual coordination. Doustan, Boveiri, Zilaei, and Seifourian (2012) clarified that in asymmetric bimanual tasks, when only the spatial direction of hands is different, a positive transfer to its converse pattern occurs. This is in contradiction to the results achieved by Vangheluwe et al. (2006). Their findings support the generalized motor program theory and the hypothesis of effector-independent motor program. However, contrary to cross-talk theory for bimanual coordination, these contradictory results imply that possibly, inter-hemisphere transfer depends on the specific features of bimanual task.

It can be inferred from different researches that effector-independent motor memory in bimanual coordination movements depend on the difference in the specific features of the movements of the two hands, which should be taken into consideration. It appears that the difference in hand movements speed and some spatial features are considered to be the features over which the dominance is difficult or presumably are not effector-independent. Whereas, if hands movements are the same in terms of temporal aspects and movement pattern and their difference is just in the direction of the movements, there will be a positive transfer and therefore, the motor program can be effector-independent. Considering the sparse research conducted on this issue, a greater need is felt for more studies in order to determine effector-independent motor features as well as effector-dependent features in bimanual coordination movements. Through conducting some researches in this field the ambiguities of bimanual coordination theories can be decreased and also criticized because some of them are in contradiction to each other. More studies on this issue, as long as there is a motor program for asymmetric bimanual movement, may be helpful in recognition of intrinsic 
aspects and generalized motor program parameters of bimanual movement. The present study intends to both investigate the transfer to its converse pattern and distinguish the effector-independent motor features by making various changes in motor patterns of the two hands (change in temporal pattern, range of motion, and load).

\section{Methods}

\section{Participants}

Study protocol was approved by the Institutional Ethics Committee. Study participants were selected from the male physical education students (30 students) of Shahid Chamran University of Ahvaz, Iran (age $21.5 \pm 1.3$ years), who were all right handed, right-eyed and with normal visual acuity (using Snellen Test). They completed a consent form before participating in the research. The personal information including gender, age, and handedness was collected from the questionnaire. Eye laterality was assessed by Hole-in-the card test. To examine the subjects' right-handedness, the Edinburgh handedness inventory made by Oldfield (1971) was used.

\section{Procedure}

The subjects were randomly divided into three groups (three groups of 10) each of which was to take a different test. Before taking the pretest, all the subjects were acquainted, by the examiner, with the test items as well as with how to draw the patterns and how to be coordinated with the sound of a metronome. The measurement tools in the present study included questionnaires to identify the personal characteristics, chronometer to determine the exact time of taking the test, two Genius G-note 7100 light pens (KYE International Corporation, Chino, CA, USA) equipped with a touch screen with a resolution of 2000 lpi connected to two laptops through USB ports, digitizing custom-made software called "Bimanual coordination apparatus for symmetrical and asymmetrical bimanual drawing" developed using MATLAB program (R2017b, MathWorks, Inc., Natick, MA, USA) and a metronome. The software had been designed in a way that the deviation from the pattern was calculated based on the number of lines deviating from specified pattern and the percentage of correct implementation of pattern was computed in digits. In three groups, the percentage of motion error was obtained by the software using the output pixels of the correct motion pattern. The total error of each test was calculated from the mean motion error of both hands.

In all three experiments, the participants first received an acquisition and a transfer pretest (converse patterns between the two hands). The exercise pattern was similar to the Acquisition test, so more difficult tasks were performed with a dominant hand and the simple task with a non-dominant hand: in the first group, load is on the right hand; in the second group, the velocity of the right hand was twice higher than on the left; in the third group, the range of motion of right hand was twice larger than of the left (see Figures 1 and 2). Transfer tests were converse patterns between the two hands. Acquisition and transfer tests were done before and after the exercise days. The exercises were performed in ten blocks of five trials in each day for four consecutive days. Each trial similar to the test could be performed at 60 beats in 30 seconds. The metronome was set on a beat in 500 milliseconds, and the participant performed a circular bimanual drawing based on this rhythm. Posttests were conducted in a similar way to the pretests. The retention test was conducted 48 hours after the fourth-day practice. The tests were carried out in simultaneous bimanual movement both as practiced pattern (acquisition) and substitution of patterns between the two hands (transfer). Each of the posttests was compared to the its same pretest. In the transfer test, if the performance of the posttest has progressed compared to the pretest, it means a positive transfer and if it has dropped, which means a negative transition.
First group

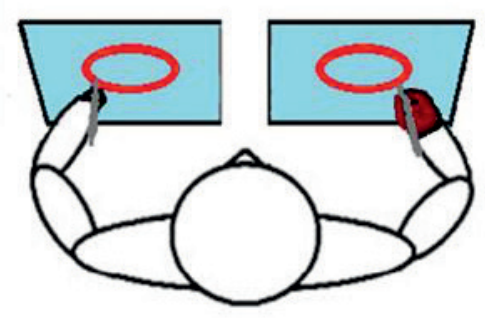

Second group

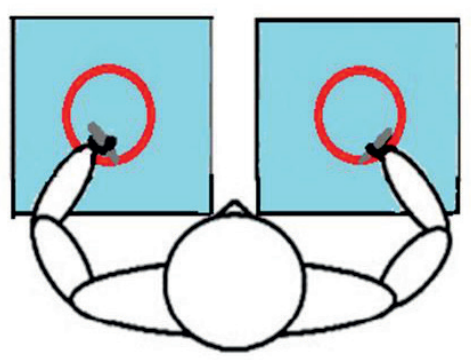

Third group

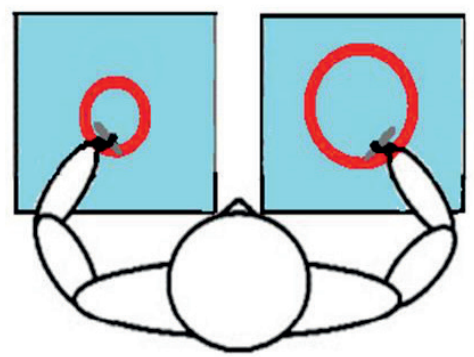

Figure 1. Pictures illustrating how to perform the exercises in three experimental groups. 

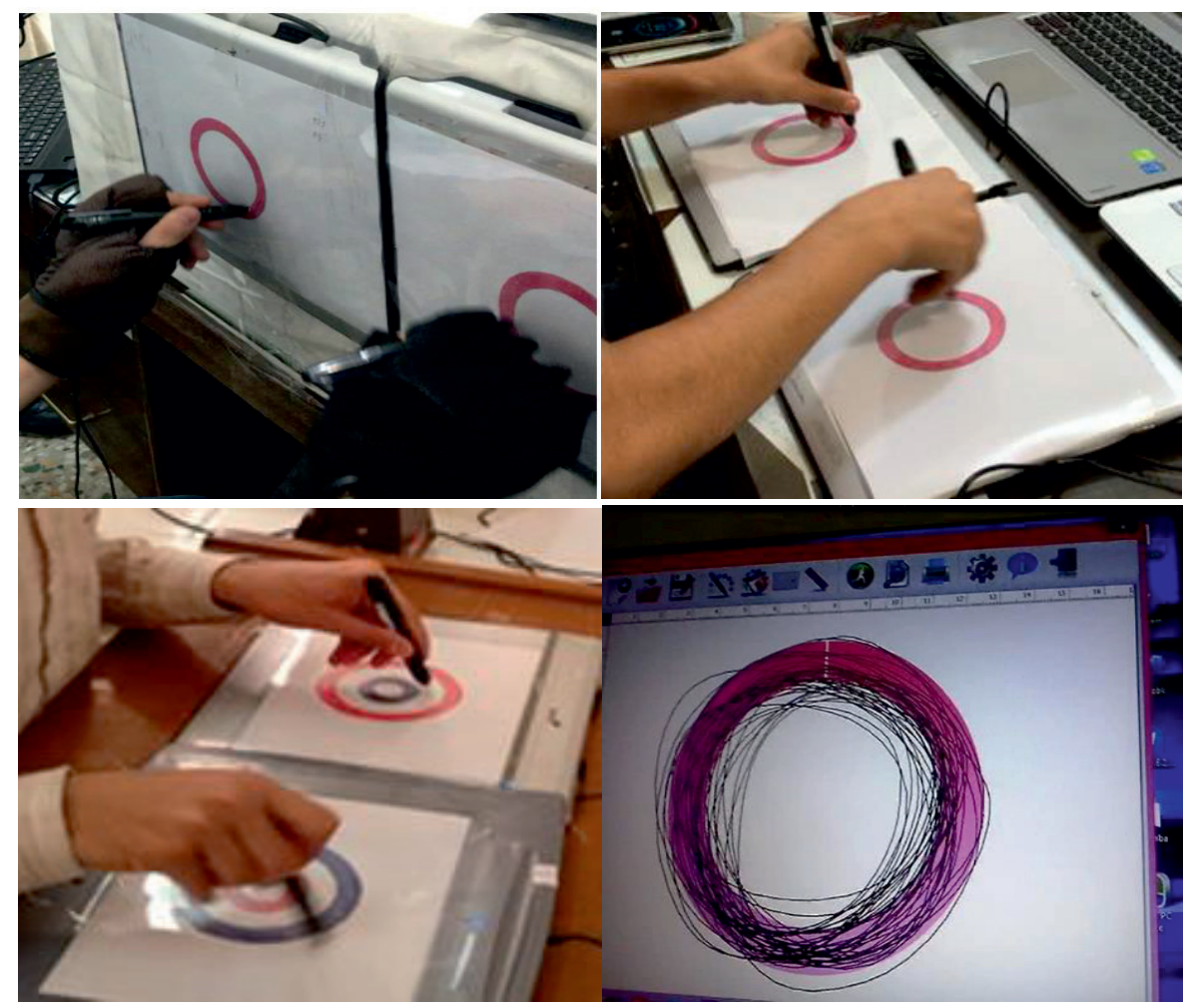

Figure 2. Pictures from implementation of bimanual movement patterns in three groups.

In the first group, we examined the bimanual task which in the effect of load on the two hands was different: the first program had been designed in such a way that the subjects of this group were supposed to draw a circle in a simultaneous bimanual task while they were wearing a pair of loose gloves on their fingers. However, there were pellets in total weight of 2 kilograms in one of the gloves (right-hand glove) which increased the effect of load compared to the other hand. According to Kennerley, Diedrichsen, Hazeltine, Semjen, and Ivry (2002) that said the easiest situation to bimanual circle drawing is the right hand draw clockwise and the left hand counterclockwise, in all groups, the right hand drew circles clockwise and left hand drew counterclockwise. The task of drawing circle was different from the other groups' tasks; that is in other groups the movements were performed in a horizontal direction, while in this group the movements were performed in a vertical direction parallel to the body (frontal). The change was implemented to assess the effect of load on the movements. Simultaneously with every beat of metronome, the circles were drawn. In the pretest, simultaneously with every beat of metronome, both hands, with and without pellets, drew circles concurrently this task was repeated 60 times during 30 seconds.

In the second group, velocity pattern difference between the two hands: in the second experiment the subjects' hands drew circle in a simultaneous bimanual task, however, they were asked to draw the circles with one hand moving twice as fast as the other with every beat of the metronome one hand (right hand) drew two clockwise circles and the other hand (left hand) drew just one counterclockwise circle. During the tests, with every beat of the metronome, the faster hand drew two circles and the slower drew one circle so that this task could be performed 30 times consecutively (the faster hand drew 60 circles over 30 seconds and the slower one rendered 30 circles).

In the third group, the difference in the difference in range of motion of the hands: the third program was designed in a way that the subjects drew circles with both hands in a simultaneous bimanual task. However, the diameter of one of the circles by one hand was to be twice larger as the other. That is with every beat of the metronome, one of the hands drew a large circle while the other drew a smaller one, concurrently with every beat this task was performed 60 times in 30 seconds.

\section{Data processing}

The data were analyzed using IBM SPSS Statistics (Version 20.0; IBM, Armonk, NY, USA). Analysis of variance for repeated measures, where dependent variable was "percentage changes of correct bimanual coordination movement" and independent variables were time 
(pretest, day1, day2, day3, day4, posttest) and group. The effect size was evaluated by the eta squared. LSD follow-up test was also employed. The significance level was considered .05 for all the tests.

\section{Results}

\section{Acquisition tests}

In within group effects test with repeated measures for asymmetric bimanual movement in three experimental groups the factor of time was significant in acquisition tests $\left(F=36.55, p<.001, \eta^{2}=.575\right)$; that is the exercises have enhanced the bimanual tasks Considering the mean percentage of correct movement, the results indicated that in all three groups, the trend of changes has inclined toward the enhancement of bimanual movements. The follow-up test revealed that there is a significant relation between the pretest phase and posttest and retention test phases in all three groups (respectively for posttest and retention test, in the first group $p=.003$, $p=.011$, in the second group $p=.001$ and $p=.001$, in the third group $p=.003$ and $p=.005)$. Nevertheless, neither a significant effect on the group $(F=1.38$, $p=.270, \eta^{2}=.092$ ) nor any interaction between the experimental group and test number $(F=1.00, p=.437$, $\left.\eta^{2}=.575\right)$ was observed. This means there has not been any significant difference in the improvements resulted from exercises between the groups.

\section{Transfer tests}

For asymmetric bimanual movements in transfer tests (when the movement of both hands are exchanged corresponding to the exercise situations), within group effects using repeated measures in three experimental groups in transfer tests, the factor of time was significant $\left(F=5.29, p<.001, \eta^{2}=.164\right)$; that is the practices have improved bimanual movements in transfer tests in general. Furthermore, significant effect on the group $\left(F=8.17, p=.002, \eta^{2}=.377\right)$ and factor*group $\left(F=2.12, p=.046, \eta^{2}=.136\right)$ was observed. Which indicates that in transfer tests, there has been a significant difference between the groups in terms of the changes resulted from exercise. The results of intragroup variance analysis with repeated measures while drawing bimanual movements in the acquisition and transfer phases for separate practice groups has been shown in Table 1. The results showed that in the first group (the group in which the effect of load on the movement of the two hands was different) there was a significant difference between the tests $\left(F=19.28, p<.001, \eta^{2}=.682\right)$. Considering the mean percentage of correct movement, the results indicated that the trend of changes in transfer tests has inclined toward the enhancement of bimanual movements. The follow-up tests in this group revealed that there is a significant difference between pretest phase and posttest and retention phases in the transfer test (respectively $p<.001, p<.001)$. Mean percentage of correct movement increased from $44 \%$ to $60 \%$ in the posttest phase and $58 \%$ in the retention phase (the trend of changes in these tests can be seen in Figure 3). The changes in the transfer test in this group are relatively similar to those in the main practiced task (acquisition tests). Nevertheless, in the second group (the group performing asymmetric bimanual movement to examine the speed of both hands) the results were different and there was no difference in the tests $\left(F=0.945, p=.432, \eta^{2}=.095\right)$. Therefore, transfer tests do not imply the enhancement of bimanual movement in its converse pattern. The follow-up tests also revealed that there is no significant difference between pretest phase and posttest and retention phases in transfer test (respectively $p=.056$ and $p=.133$ ) although mean percentage of correct

Table 1

The results of intragroup variance analysis with repeated measures (factor time) while drawing with bimanual movements in acquisition and transfer phases for separate practice groups

\begin{tabular}{lccc}
\hline & $F$ & $p$ & $\eta^{2}$ \\
\hline Acquisition & & & \\
$\quad$ First group & 8.92 & $<.001$ & .498 \\
Second group & 11.31 & $<.001$ & .558 \\
Third group & 18.97 & $<.001$ & .678 \\
Transfer & & & \\
First group & 19.28 & $<.001$ & .682 \\
Second group & 0.945 & .432 & .095 \\
Third group & 4.91 & .005 & .353 \\
\hline
\end{tabular}


movement increased from $34 \%$ to $42 \%$ in posttest and to $42 \%$ in retention test (the trend of changes in these tests can be seen in Figure 4). In the third group (the group performing asymmetric bimanual movement to examine the range of motion of both hands) there was a considerable difference compared to other groups in transfer test. Mean percentage of correct movement increased from $41 \%$ to $66 \%$ in posttest phase and $64 \%$ in the retention phase in as much as there was a significant difference between the tests when drawing the asymmetric bimanual task in this group in transfer phase immediately after the exercises and transfer in retention phase $\left(F=4.91, p=.005, \eta^{2}=.353\right)$. Considering the mean percentage of correct movement, the results indicated that the changes in transfer tests have brought about decline in bimanual movements and have led to the failure of performance. Although the follow-up tests showed, that despite the decline in performance, the difference between the pretest phase and posttest phase is not statistically significant, this difference is significant between the pretest phase and retention phase in transfer test ( $p=.047, p=.180$ respectively) it indicates a negative transfer. The mean percentage of correct movement decreased from $53 \%$ to $51 \%$ in posttest phase and $50 \%$ in retention phase in the transfer test (the trend of changes in these tests can be seen in Figure 5).

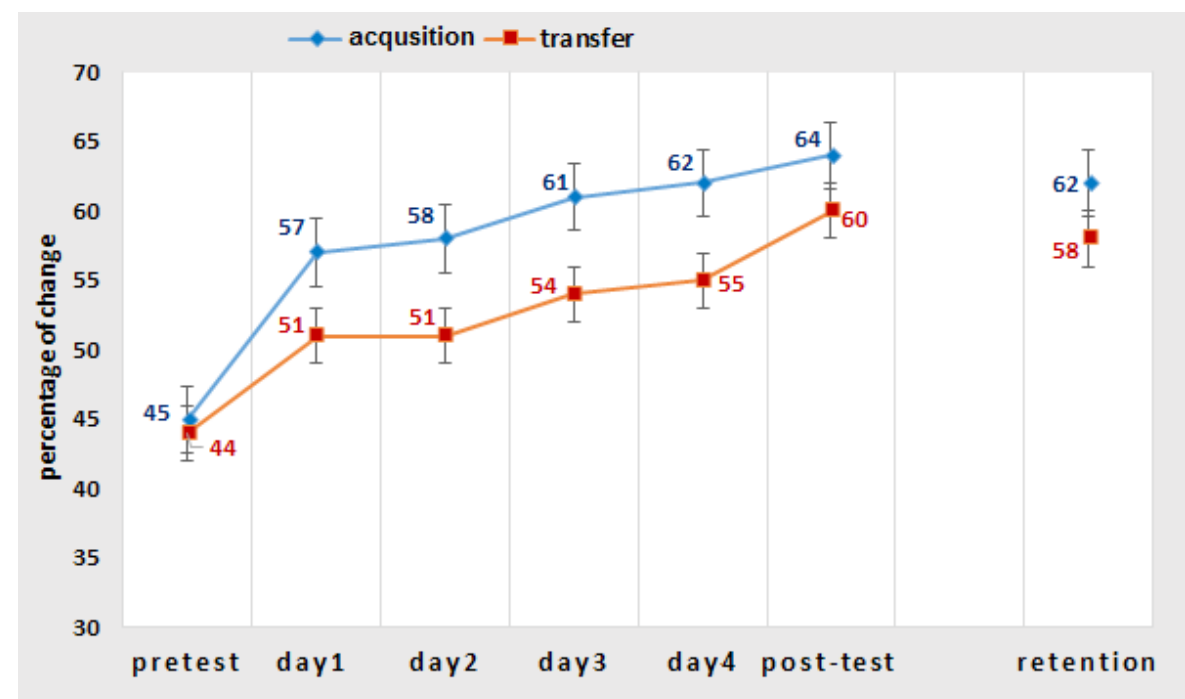

Figure 3. Percentage changes of correct bimanual coordination movement in acquisition and transfer tests in the first group.

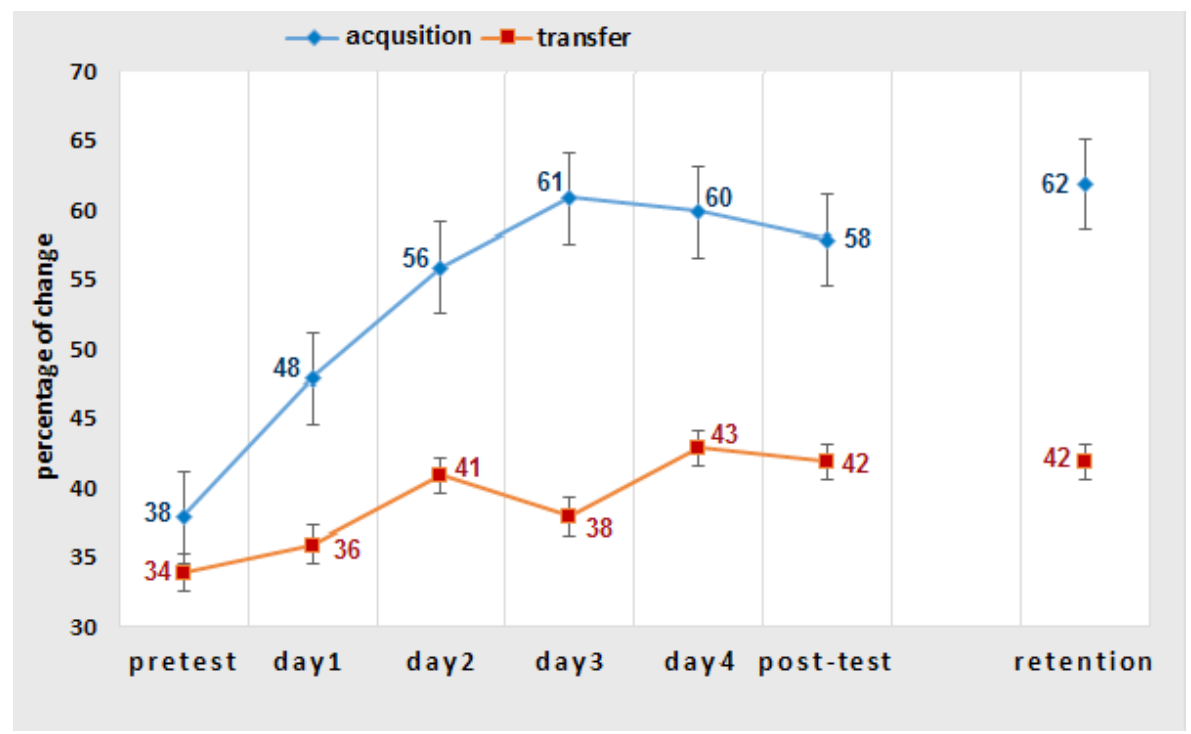

Figure 4. Percentage changes of correct bimanual coordination movement in acquisition and transfer tests in the second group. 


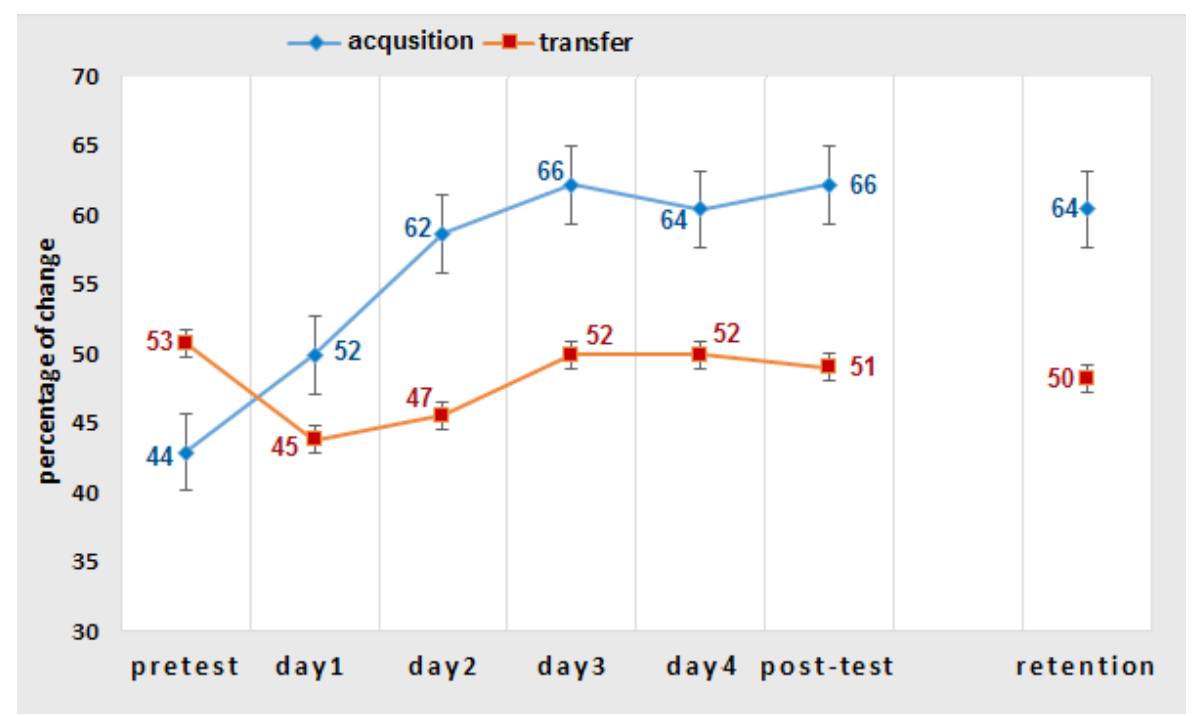

Figure 5. Percentage changes of correct bimanual coordination movement in acquisition and transfer tests in the third group.

\section{Discussion}

The results of the study showed that there was no significant difference among the three groups in the acquisition tests, so those that had trained all learned bimanual movement well. But in the transfer tests, a remarkable difference was observed among the groups that our interpretations of the bimanual movement were affected.

The findings of the first group showed that in the exercise in which the effect of load on the movement of the two hands is different, transfer of asymmetric bimanual movement to the exchanged position between the two hands is positive. Increasing the load increases the effect of gravity on the hand. The results of Bringoux, Blouin, Coyle, Ruget, and Mouchnino (2012) showed that loading on the hand and changing the gravity effect on the hands motor command and thus the motor performance. They stated that loading on the hand, changing firing muscle spindles during active contraction and sense of movement, results in significant changes in movement accuracy. The force of gravity has a significant effect on spatial orientation, proprioception, exchange, and initiation or stop of our movements. The CNS can encounter gravity in one of the two levels of programming or performance (Papaxanthis, Pozzo, \& McIntyre, 1998). Papaxanthis et al. (1998) state that in programming level, gravity is not considered to be only a simple force to be overcome rather it is an influential factor which could be used for performing the movements. On the performance level, the CNS copes with gravity impetus along with motion and based on the data resulted from proprioception. Considering gravity as a disruptive mechanical agent impedes assessment of gravity impetus before the action and consequently considerably affects the accuracy of the movement (Papaxanthis et al., 1998).

Although, mechanically, gravity-related constraints can affect the movements of the hands (Nishikawa, Murray, \& Flanders 1999; Soechting \& Flanders, 1998) and some have suggested that the force of gravity may contribute to the development of motor program (Papaxanthis, Pozzo, \& McIntyre 2005; Papaxanthis, Pozzo, \& Schieppati 2003), considering that in the present study, transfer to the exchange of movements between the two hands is positive, it appears that overcoming the force of gravity (in the present research the weights attached to the hands) in bimanual coordination, is controlled at the performance level and lower levels of the nervous system and overcoming these constraints in bimanual coordination does not need changing the motor program formed before and probably, this feature is controlled by the mechanism of the muscular spindle and in the reflexive level, does not engender much interference between the two hands. Thus, it is not considered to be one of the main features of the bimanual motor program. So, like movement direction it can be easily transferred to bimanual movement in converse situations (exchanged between the two hands). The results of the research in the first group support the studies which have been conducted by Zanone and Kelso $(1992,1997)$. Their research showed that previously existing bimanual coordination tendencies were exploited during learning in order to match visual requirements. These findings and the concepts presented provide a framework for understanding how learning occurs in the context of previous 
experience. The results of the study in the second and third group in which the movement difference of the two hands in terms of speed and range of motion were examined, revealed different findings compared to the first two groups inasmuch as in the second group no transfer to converse pattern occurred and in the third group the results were even more noticeable and transfer to the exchange of patterns between the hands was negative. The results of the transfer of asymmetric bimanual movement in these two groups markedly reinforces the research which has been conducted by Vangheluwe et al. (2006) and Sisti et al. (2011). These results indicate considerably important points which can challenge any of the theories proposed on bimanual coordination. Vangheluwe et al. (2006) reported that in bimanual finger tapping with a 2:1 ratio, transfer to converse pattern is negative. Sisti et al. (2011) concluded in his research that isofrequency bimanual patterns (1:1) were performed more successfully than multifrequency patterns (1:3, 1:2 and 2:3). They also stated that the transfer of this multi-frequency movement to the inverse pattern is difficult.

According to hierarchical control theory, the main properties or intrinsic aspects of bimanual coordination motor program (if there is any for bimanual movements) are controlled at a high level of the nervous system control hierarchy and variable aspects related to the conditions of environment and task are controlled in low levels of hierarchy (Danion \& Latash, 2011; Diedrichsen, Shadmehr, \& Ivry, 2010). It turns out that movement direction of the two hands (studied in previous researches) in addition to the effect of load on bimanual coordination are among the taskrelated properties and are the parameters controlled in low levels and can be exchanged easily between the two hands. The results of Muehlbauer, Panzer, and Shea (2007) indicated that participants were able to effectively compensate for decreased and increased load with virtually no changes in performance characteristics (displacement, velocity, acceleration, and pattern of durations) while electromyographic signals demonstrated that smaller (reduced load) or larger forces (increased load) were spontaneously generated to compensate for the change in load. Dimitriou and Buckingham (2018) found that in bimanual task no changes in holding force as a direct consequence of the other hand's actions. Their results also suggest that fingertip forces are largely independent between hands in a bimanual lifting context but are sensitive to different task demands.

While the properties such as size and temporal difference of the movements of two hands during asymmetric bimanual movements are considered to be intrinsic properties (or invariable aspects) of bimanual movements, and are controlled at a high level of the central nervous system. Therefore, depending on the hierarchical level at which, the difference in the two hands is controlled, it appears that transfer of asymmetric bimanual movement to exchanged pattern between the two hands can be positive or negative. Considering the results of the third group that confirmed negative transfer and even the results of the second group which did not end in positive transfer, discussion on generalized motor program theory can cover remarkable angles and introduce new subjects in bimanual movements. According to GMP, asymmetric bimanual coordination movements should be regarded from a different angle coupling between two hands should be considered as a primary motor program which has itself invariable aspects and in lower levels some parameters are added to that (Ivry, Diedrichsen, Spencer, Hazeltine, \& Semjen, 2004). Considering the results, some of these parameters could be brought up in the present research.

Neurologically, in bimanual movements, a good majority of the research has referred to the outstanding role of motor cortex of the dominant hemisphere (Cardoso de Oliveira, 2002; Nozaki \& Scott, 2009) and specifically motor cortex in the dominant hemisphere (Makia, Wonge, Sugiuraa, Ozakic, \& Sadatoa, 2008). Nevertheless, in all cortex areas, the role of supplementary motor area in bimanual coordination especially in asymmetric bimanual movements has been emphasized (Goerres, Samuel, Jenkins, \& Brooks, 1998; Toyokura, Muro, Komiya, \& Obara, 1999). Implementing asymmetric bimanual motor patterns involves a high level of calculation in nervous system and includes parietal cortex (Wenderoth, Debaere, Sunaert, van Hecke, \& Swinnen, 2004) and probably the more difficult the bimanual task is, a more computational attempt is needed. Meister, Foltys, Gallea, and Hallet (2010) showed that the control process of bimanual movements in which the two hands are temporally and spatially asymmetric (amplitude of the movement), engage higher level areas of dorsolateral prefrontal cortex and anterior cingulate cortex. They maintain that activation of these areas is probably related to the movement performance pattern and difficulty of the task (Meister et al., 2010). Similarly, Wenderoth, Debaere, Sunaert, and Swinnen (2005) showed that the interaction between parietal-premotor network and the dorsolateral prefrontal cortex to overcome the spatial interaction in bimanual movements (asymmetry in the amplitude of movement of the two hands) is essential. Rueda-Delgado et al. (2014) hold that in first phases of bimanual coordination, greater areas of brain are stimulated, whereas in the final phases after sufficient practice, spatial pattern of nervous activity can decrease due to neuron reorganization. 


\section{Conclusion}

In total, it can be concluded that difficulty levels or difference of various levels of cerebral control in these two bimanual tasks (difference in speed and amplitude of movement of the two hands) compared to the first task (difference in the effect of load on the two hands) is bigger, which can reinforce the conclusion that asymmetry in temporal pattern and amplitude of movement of the two hands is the integral part of bimanual motor program (presumably it can be regarded as an intrinsic property of bimanual motor program) which cannot be transferred to the exchanged pattern between the two hands.

The results showed that in asymmetric bimanual tasks, the transfer to the inverse pattern can be positive or negative. So that some of the movement properties are transferable and not some others. On the whole, depending on which hierarchical level of bimanual motor program control includes difference in asymmetric pattern of the two hands among its control characteristics, it may or may not be transferred to its converse pattern. Therefore, the theory of the independence of effector from bimanual coordination motor program needs revision. Additionally, the results of the present research might persuade us that bimanual coordination motor program like other uni-limb motor programs can hold intrinsic features and parameters which change according to the task features and environment.

\section{Conflict of interest}

There were no conflicts of interest.

\section{References}

Bangert, S. A., Reuter-Lorenz, P. A., Walsh, C. M., Schachter, A. B., \& Seidler R. D. (2010). Bimanual coordination and aging: Neurobehavioral implications. Neuropsychologia, 48, 1165-1170.

Bringoux, L., Blouin, J., Coyle, T., Ruget, H., \& Mouchnino, L. (2012). Effect of gravity-like torque on goal-directed arm movements in microgravity. Journal of Neurophysiology, 107, 2541-2548.

Cardoso de Oliveira, S. (2002). The neuronal basis of bimanual coordination: Recent neurophysiological evidence and functional models. International Journal of Psychonomics, 110, 139-159.

Danion, F., \& Latash, M. L. (2011). Motor control: Theories, experiments, and applications. Oxford, United Kingdom: Oxford University Press.

Diedrichsen, J., Shadmehr, R., \& Ivry, R. B. (2010). The coordination of movement: Optimal feedback control and beyond. Trends in Cognitive Sciences, 14, 31-39.
Dimitriou, P., \& Buckingham, G. (2018). Bimanual lifting: Do fingertip forces work independently or interactively? Journal of Motor Behavior, 50, 26-36.

Doustan, M., Boveiri, K., Zilaei, B., \& Seifourian, M. (2012). Barresiye enteghale harekate dodastiye namotagharen be halite akse an: tahlili bar nazariyehaye harekate dodasti [The study of transfer of asymmetrical bimanual movement to its converse pattern: Analysis on bimanual movements theories]. Journal of Motor Behavior and Sport Psychology, 1, 553-564.

Goerres, G. W., Samuel, M., Jenkins, H., \& Brooks, D. J. (1998). Cerebral control of unimanual and bimanual movements: An $\mathrm{H}_{2}{ }^{15} \mathrm{O}$ PET study. NeuroReport, 9, 3631-3638.

Ivry, R., Diedrichsen, J., Spencer, R., Hazeltine, E., \& Semjen, A. (2004). A cognitive neuroscience perspective on bimanual coordination and interference. In S. P. Swinnen \& J. Duysens (Eds.), Neuro-behavioral determinants of interlimb coordination (pp. 259-295). Boston, MA: Kluwer Academic.

Kennerley, S. W., Diedrichsen, J., Hazeltine, E., Semjen, A., \& Ivry, R. B. (2002). Callosotomy patients exhibit temporal and spatial uncoupling during continuous bimanual movements. Nature Neuroscience, 5, 376-381.

Klapp, S. T., Nelson, J. M., \& Jagacinski, R. J. (1998). Can people tap concurrently bimanual rhythms independently? Journal of Motor Behavior, 30, 301-322.

Kurtz, S., \& Lee, T. D. (2003). Part and whole perceptualmotor practice of a polyrhythm. Neuroscience Letters, 338, 205-208.

Makia, B. Y., Wonge, K. F., Sugiuraa, M., Ozakic, T., \& Sadatoa, N. (2008). Asymmetric control mechanisms of bimanual coordination: An application of directed connectivity analysis to kinematic and functional MRI data. NeuroImage, 42, 1295-1304.

Marteniuk, R. G., \& MacKenzie, C. L. (1980). Information processing in movement organization and execution. In R. Nickerson (Ed.), Attention and performance VIII (pp. 29-57). Hillsdale, NJ: Erlbaum.

Meister, I. G., Foltys, H., Gallea, C., \& Hallett, M. (2010). How the brain handles temporally uncoupled bimanual movements. Cerebral Cortex, 20, 2996-3004.

Muehlbauer, T., Panzer, S., \& Shea, C. H. (2007). The transfer of movement sequences: Effects of decreased and increased load. Quarterly Journal of Experimental Psychology, 60, 770-778.

Nishikawa, K. C., Murray, S. T., \& Flanders, M. (1999). Do arm postures vary with the speed of reaching? Journal of Neurophysiology, 81, 2582-2586.

Nozaki, D., \& Scott, S. H. (2009). Multi-compartment model can explain partial transfer of learning within the same limb between unimanual and bimanual reaching. Journal of Experimental Brain Research, 194, 451-463.

Oldfield, R. C. (1971). The assessment and analysis of handedness: The Edinburgh Inventory. Neuropsychologia, 9, 97-113.

Papaxanthis, C., Pozzo, T., \& McIntyre, J. (1998). Arm endpoint trajectories under normal and micro-gravity environments. International Academy of Astronautics, 43, 153-161.

Papaxanthis, C., Pozzo, T., \& McIntyre, J. (2005). Kinematic and dynamic processes for the control of pointing 
movements in humans revealed by short-term exposure to microgravity. Neuroscience, 35, 371-383.

Papaxanthis, C., Pozzo, T., \& Schieppati, M. (2003). Trajectories of arm pointing movements on the sagittal plane vary with both direction and speed. Experimental Brain Research, 148, 498-503.

Rueda-Delgado, L. M., Solesio-Jofre, E. A., Serrien, D. J., Mantini, D., Daffertshofer, A., \& Swinnen, S. P. (2014). Understanding bimanual coordination across small time scales from an electrophysiological perspective. Neuroscience \& Biobehavioral Reviews, 47, 614-635.

Schmidt, R. A., \& Lee, T. D. (1999). Motor control and learning: A behavioral emphasis (3rd ed.). Champaign, IL: Human Kinetics.

Sisti, H. M., Geurts, M., Clerckx, R., Gooijers, J., Coxon, J. P., Heitger, M. H., ... Swinnen, S. P. (2011). Testing multiple coordination constraints with a novel bimanual visuomotor task. PLOS ONE, 6, e23619.

Soechting, J. F., \& Flanders, M. (1998). Movement planning: Kinematics, dynamics, both or neither? In L. R. Harris \& M. Jenkin (Eds.), Vision and action (pp. 352-371). Cambridge, United Kingdom: Cambridge University Press.

Swinnen, S. P. (2002). Intermanual coordination: From behavioural principles to neural-network interactions. Nature Reviews Neuroscience, 3, 348-359.

Swinnen, S. P., \& Carson, R. G. (2002). The control and learning of patterns of interlimb coordination: Past and present issues in normal and disordered control. International Journal of Psychonomics, 110, 129-137.

Swinnen, S. P., Dounskaia, N., \& Duysens, J. (2002). Pattern of bimanual interference reveal movement encoding within a radial egocentric reference frame. Journal of Cognitive Neuroscience, 14, 463-471.

Swinnen, S. P., Jardin, K., Meulenbroek, R., Dounskaia, N., \& Hofkens-Van Den Brandt, M. (1997). Egocentric and allocentric constraints in the expression of patterns of interlimb coordination. Journal of Cognitive Neuroscience, 9, 348-377.

Toyokura, M., Muro, I., Komiya, T., \& Obara, M. (1999). Relation of bimanual coordination to activation in the sensorimotor cortex and supplementary motor area: Analysis using functional magnetic resonance imaging. Brain Research Bulletin, 48, 211-217.

Vangheluwe, S., Suy, E., Wenderoth, N., \& Swinnen, S. P. (2006). Learning and transfer of bimanual multifrequency patterns: Effector-independent and effector-specific levels of movement representation. Experimental Brain Research, 170, 543-554.

Walsh, R. R., Small, S. L., Chen, E. E., \& Solodkin, A. (2008). Network activation during bimanual movements in humans. NeuroImage, 43, 540-553.

Wenderoth, N., Debaere, F., Sunaert, S., \& Swinnen, S. P. (2005). Spatial interference during bimanual coordination: Differential brain networks associated with control of movement amplitude and direction. Human Brain Mapping, 26, 286-300.

Wenderoth, N., Debaere, F., Sunaert, S., van Hecke, P., \& Swinnen, S. P. (2004). Parieto-premotor areas mediate directional interference during bimanual movements. Cerebral Cortex, 14, 1153-1163.

Wu, T., Wang, L., Hallett, M., Li, K., \& Chan, P. (2010). Neural correlates of bimanual anti-phase and in-phase movements in Parkinson's disease. Brain, 133, 2394-2409.

Zanone, P. G., \& Kelso, J. A. (1992). Evolution of behavioral attractors with learning: Nonequilibrium phase transitions. Journal of Experimental Psychology, 18, 403-421.

Zanone, P. G., \& Kelso, J. A. (1997). Coordination dynamics of learning and transfer: Collective and component levels. Journal of Experimental Psychology, 23, 1454-1480. 